\title{
Integrating Shape and Color Features for Adaptive Real-time Object Tracking
}

\author{
Junqiu WANG and Yasushi YAGI \\ The institute of Scientific and Industrial Research, Osaka University \\ 8-1 Mihogaoka, Ibaraki, Osaka, 560-0047 \\ Email: jerywang@public3.bta.net.cn, yagi@am.sanken.osaka-u.ac.jp
}

\begin{abstract}
We extend the standard mean shift tracking algorithm to an adaptive tracker by selecting reliable features from color and shape cues. The standard mean shift algorithm assumes that the representation of tracking targets is always sufficiently discriminative enough against background. Most tracking algorithms developed based on the mean shift algorithm use only one cue (such as color) throughout their tracking process. The widely used color features are not always discriminative enough for target localization because illumination and viewpoint tend to change. Moreover, the background may be of a color similar to that of the target. We present an adaptive tracking algorithm that integrates color and shape features. Good features are selected and applied to represent the target according to the descriptive ability of these features. The proposed method has been implemented and tested in different kinds of image sequences. The experimental results demonstrate that our tracking algorithm is robust and efficient in the challenging image sequences.
\end{abstract}

\section{INTRODUCTION AND RELATED WORK}

Real-time object tracking plays an important role in many applications such as robotics [18], visual surveillance [4], human computer interface [1], [2], [10], and intelligent transportation systems [11]. Much effort has been made and progress has been achieved in detection and tracking of object in image sequences. However, object tracking remains an unsolved problem because of several difficulties. For example, tracking may drift away from a target due to background or viewpoint changes. Different illumination conditions, occlusions, and sudden motion bring more trouble to the tracking.

Model-based [9] and appearance-based [6] approaches have been investigated for solving the tracking problem. Appearance-based approaches are more practical for many applications since they do not have to make 3-D models which are both difficult and expensive to obtain. Most appearance-based tracking approaches are based on some kind of representation of image appearance. Object template [12], subspace models [3], two frame flow estimation [13], [14] and motion-compensated images [16] are the commonly investigated approaches. Target representation and localization are crucial in the robustness and efficiency of the tracker [6]. The mean shift tracking algorithm [6] provides an elegant framework for target representation and localization.

The mean shift algorithm has achieved considerable success in object tracking due to its simplicity and robustness.
It finds local minima of a similarity measure between the color histograms (or kernel density estimations) of the model and the candidates in the image. The mean shift tracking algorithm is based on an appearance model. Different kinds of color features have been widely used due to their simplicity and low computational cost [6], [10]. They are reliable for many tracking tasks, even when there is partial occlusion, small deformation or overlap. Color histogram is the most popular color feature that has been used to represent the appearance of the target. However, color features cannot give good performance when the representation of the tracked object and background have similar color. The color of an object depends on illumination, viewpoint and camera parameters that tend to change during a long tracking. Moreover, the color histograms omit the shape of the target which is very helpful for the tracking. Thus the static color features are not always discriminative enough.

To cope with the problems mentioned above, one can improve the robustness by using an adaptive tracking scheme. The basic idea is to adaptively select features that make the object discriminative against the background. Stern and Efros [10] gave an adaptive tracking algorithm in which the best feature is chosen from 5 color spaces. Collins and Liu [4] have proposed an adaptive tracker that can select the discriminative color features according to the discriminative ability of features. The set of candidate color features they used includes 49 features that are calculated by linearly combining R, G and B pixel values. Since they employ a large set of candidate features, it is computationally expensive to select the appropriate features. In addition, only color features are used, which may not be discriminative enough under certain circumstances.

It has been known that multi-cue plays a powerful role in human visual perception. While using multiple cues in detection or tracking, two sets of features should be complementary. In other words, when one cue fails the other one can deal with the difficulty. Although the color cue is very important in detection and tracking, other types of cues such as shape can be very helpful especially when they are integrated with color. The shape cue can help color conquer these difficulties and so does color cue [1]. In this paper, we propose an adaptive tracking algorithm in which the best features are selected from the shape and color cues. The shape cue is represented by gradient 
orientation histograms and the color cue is described by color histograms. We calculate color histograms in the RGB, the HSV spaces and the normalized $r g$ space. The most discriminative features are selected by evaluating the discriminative ability of each feature. The evaluation is done by calculating the variance of the mutual information. The mutual information measures the statistical dependence between two variables. Once the best features have been selected, the tracker uses these new features to update the tracking model.

The paper is organized as follows. In Section II, we introduce how to extract the color and shape features to represent the target. Section III discusses the feature selection method. The adaptive tracking method of the target in a new frame is given in Section IV. We evaluate the performance of the proposed method in Section V. This paper is concluded in Section VI.

\section{Feature EXtraction}

\section{A. Color Cue}

Color distributions are represented by the color histograms. We calculate the color histograms in three color spaces: the RGB space, the HSV space and the normalized $r g$ space. The R,G and B channels are quantized into 12 bins respectively. The RGB space is not enough for discrimination under all circumstances. The RGB space is converted into the HSV space and both hue and saturation are quantized into 12 bins. We found that intensity is less helpful in our tracking tasks. Thus the intensity factor is not used here. The $r g$ space has been shown to be reliable when the illumination changes. Thus $r$ and $g$ are also employed. There are 7 color features in the candidate feature set.

Color histogram is calculated by using a weighting scheme. Different pixel's contribution to the object representation depends on its position with respect to the center of the target. Pixels near the region center are more reliable than those further away. Smaller weights are given to those further pixels by using Epanechnikov kernel [6] as a weighting function:

$$
k(\mathbf{x})=\left\{\begin{array}{c}
\frac{1}{2} c_{d}^{-1}(d+2)\left(1-\|\mathbf{x}\|^{2}\right), \text { if }\|\mathbf{x}\|^{2} \leq 1 \\
0, \text { otherwise }
\end{array}\right.
$$

where $c_{d}$ is the volume of the unit $d$-dimensional sphere. Thus, we increase the reliability of the color distribution when these boundary pixels belong to the background or get occluded.

The color distribution $h_{f}=\left\{p_{f}^{\left(b_{i n}\right)}\right\}_{b_{i n}=1 \ldots m}$ of the target is given by

$$
p_{f}^{\left(b_{i n}\right)}=C_{f} \sum_{\mathbf{x}_{i} \in R_{f}} k\left(\left\|\mathbf{x}_{i}\right\|\right) \delta\left[h\left(\mathbf{x}_{i}\right)-b_{i n}\right],
$$

where $\delta$ is the Kronecker delta function and $h\left(\mathbf{x}_{i}\right)$ assigns one of the $m$-bins ( $m=12$ ) of the histogram to a given color at location $\mathbf{x}_{i} . C_{f}$ is normalization constant. It is calculated as

$$
C_{f}=\frac{1}{\sum_{\mathbf{x}_{i} \in R_{f}} k\left(\left\|\mathbf{x}_{i}\right\|^{2}\right)} .
$$

The tracking algorithm searches for the target in a new frame from the target candidates. The target candidates are represented by

$$
p_{c}^{\left(b_{i n}\right)}(\mathbf{y})=C_{b} \sum_{\mathbf{x}_{i} \in R_{f}} k\left(\frac{\left\|\mathbf{y}-\mathbf{x}_{i}\right\|}{h}\right)^{2} \delta\left[h\left(\mathbf{x}_{i}\right)-b_{i n}\right],
$$

where $C_{b}$ is

$$
C_{b}=\frac{1}{\sum_{\mathbf{x}_{i} \in R_{c}} k\left(\left\|\frac{\mathbf{y}-\mathbf{x}_{i}}{h}\right\|\right)^{2}} .
$$

\section{B. Shape Cue}

The shape cue is described by orientation histograms. To build a orientation histogram, we have to calculate image derivatives in $x$ and $y$ directions. We did not use the popular Sobel masks in this calculation. Instead, the Scharr masks $\left(S_{x}\right.$ and $\left.S_{y}\right)$ are employed here because it gives more accurate results than the Sobel kernel.

The gradients at the point $(x, y)$ in the image $I$ can be calculated by convolving the Scharr masks with the image:

$$
D_{x}(x, y)=S_{x} * I(x, y),
$$

and

$$
D_{y}(x, y)=S_{y} * I(x, y) .
$$

The strength of the gradient at the point $(x, y)$

$$
D(x, y)=\sqrt{D_{x}(x, y)^{2}+D_{y}(x, y)^{2}} .
$$

In order to ignore noise, a threshold is given

$$
D^{\prime}(x, y)=\left\{\begin{array}{c}
D(x, y), \text { if } D(x, y) \geq T_{D}, \\
0, \text { otherwise },
\end{array}\right.
$$

where $T_{D}$ is a threshold given empirically.

The orientation of the edge is

$$
\theta(x, y)=\arctan \left(\frac{D_{y}(x, y)}{D_{x}(x, y)}\right) .
$$

The orientations are also quantized into 12 bins. A orientation histogram can be calculated using a approach similar to the calculation of color histogram introduced in the previous subsection.

\section{FEATURE SELECTION}

\section{A. Likelihood Ratio}

The weighted histograms introduced in Section II do not directly reflect the descriptive ability of the features. A log-likelihood ratio histogram can help to solve this problem [4], [15]. We get log-likelihood ratios based on the histograms of foreground and background with respect to a given feature. The likelihood ratio produces a function that maps the feature values associated with the target to 
positive values and the background to negative values. The frequency of the pixels appeared in a histogram bin is calculated as

$$
\zeta_{f}^{\left(b_{i n}\right)}=\frac{p_{f}^{\left(b_{i n}\right)}}{n_{f g}}
$$

and

$$
\zeta_{b}^{\left(b_{i n}\right)}=\frac{p_{b}^{\left(b_{i n}\right)}}{n_{b g}},
$$

where $n_{f g}$ is the pixel number of the target region and $n_{f g}$ the pixel number of the background.

The log-likelihood ratio for a feature value $i$ is given by

$$
L^{\left(b_{i n}\right)}=\max \left(-1, \min \left(1, \log \frac{\max \left(\zeta_{f}^{\left(b_{i n}\right)}, \delta_{L}\right)}{\max \left(\zeta_{b}^{\left(b_{i n}\right)}, \delta_{L}\right)}\right),\right.
$$

where $\delta_{L}$ is a very small number. The likelihood image for each feature is created by back-projecting the ratio into each pixel in the image.

Likelihood ratio images are the foundation for evaluating the discriminative ability of the features in the candidate feature set. In Fig.1, the likelihood ratio images of different features are shown.

\section{B. Feature Selection}

Given $m_{d}$ features for tracking, the purpose of the feature selection module is to find the best subset feature of size $m_{m}$, and $m_{m}<m_{d}$. Feature selection can help minimize the tracking error and maximize the discrimination ability of the feature set. The mutual information theory can be used here to select good features which can make the target to be discriminative against the background. The mutual information is the relative entropy between the joint distribution of the target and the background and the product of the distributions. It is defined as

$$
M(F, B)=H(F)+H(B)-H_{j}(F, B),
$$

where $H(F)$ and $H(B)$ are the entropies of the foreground (target) and the background respectively, $H_{j}$ is the joint entropy of the foreground and the background. The mutual information is minimized when the feature is the most discriminative. Mutual information is very expensive to evaluate directly. Instead of directly ranking the features with the mutual information, we find the features with the largest corresponding variances of the mutual information. Following the method in [4], based on the equality $\operatorname{var}(x)=E x^{2}-(E x)^{2}$, the variance of Equation(10) is computed as

$$
\operatorname{var}(L ; p)=E\left[\left(L^{b_{i n}}\right)^{2}\right]-\left(E\left[L^{b_{i n}}\right]\right)^{2} .
$$

The variance ratio of the likelihood function is defined as [4]:

$$
\mathrm{VR}=\frac{\operatorname{var}(B \cup F)}{\operatorname{var}(F)+\operatorname{var}(B)}=\frac{\operatorname{var}\left(L ;\left(p_{f}+p_{b}\right) / 2\right)}{\operatorname{var}\left(L ; p_{f}\right)+\operatorname{var}\left(L ; p_{b}\right)}
$$

We evaluate the discriminative ability of each feature by calculating the variance ratio. In the candidate feature set, the color feature includes 7 different features: color histograms of $\mathrm{R}, \mathrm{G}, \mathrm{B}, \mathrm{H}, \mathrm{S}, r$, and $g$; and the shape feature includes gradient orientation histogram. These features are ranked according to the discriminative ability by comparing the variance ratio. The feature with the maximum variance ratio is taken as the most discriminative feature. In Fig.1, the shape feature and color feature G are ranked as the top two features, and followed by color feature B, R, S, H, $r$, and $g$.

\section{Adaptive Mean Shift Tracking}

\section{A. The Standard Mean Shift Tracking Algorithm}

The mean shift algorithm is a robust non-parametric probability density estimation method for climbing density gradients to find the mode of probability distributions of samples. It can estimate the density function directly from the data without any assumptions about the underlying distribution. This virtue avoids having to choose a model and estimating its distribution parameters [5]. The algorithm has achieved great success in object tracking [6] and image segmentation [5]. However, the basic mean shift tracking algorithm assumes that the target representation is discriminative enough against the background. This assumption is not always true especially when tracking is carried out in a dynamic background, e.g., surveillance with a moving camera. We extend the basic mean shift algorithm to an adaptive mean shift tracking algorithm which can choose the most discriminative features for effective tracking.

The standard mean shift tracker finds the location corresponding to the target in the current frame based on the appearance of the target. Therefore, a similarity measure is needed between the color distributions of a region in the current frame and the target model. A popular measure between two distributions is the Bhattacharyya distance [6], [8]. Considering discrete densities such as two color histograms $p=\left\{p^{(u)}\right\}_{u=1 \ldots m}$ and $q=\left\{q^{(u)}\right\}_{u=1 \ldots m}$ the coefficient is calculated by:

$$
\rho[p, q]=\sum_{b_{i n}=1}^{m} \sqrt{p^{\left(b_{i n}\right)} q^{\left(b_{i n}\right)}}
$$

The larger $\rho$ is, the more similar the distributions are. For two identical histograms we obtain $\rho=1$, indicating a perfect match. As distance between two distributions the measure can be defined as [6]:

$$
d=\sqrt{1-\rho[p, q]},
$$

which $d$ is the Bhattacharyya distance.

The tracking algorithm is to recursively compute of an offset value from the current location $\hat{\mathbf{y}}_{0}$ to a new location $\hat{\mathbf{y}}_{1}$ according to the mean shift vector. $\hat{\mathbf{y}}_{1}$ [5], [6] is calculated by using

$$
\hat{\mathbf{y}}_{1}=\frac{\sum_{i=1}^{n_{h}} x_{i} w_{i} g\left(\frac{y-x_{i}}{h}\right)}{\sum_{i=1}^{n_{h}} w_{i} g\left(\frac{y-x_{i}}{h}\right)} .
$$


where $w_{i}=\sum_{u=1}^{m} \sqrt{\frac{q^{(u)}}{p^{(u)}\left(\mathbf{y}_{0}\right)}} \delta\left[h\left(\mathbf{x}_{i}\right)-b_{i n}\right]$ and $g(x)=$ $-k^{\prime}(x)$.

\section{B. The Number of Features for Adaptive Tracking}

After the evaluation of the features in the candidate set, these features are ranked according to their discriminative ability against the background. The features with good discriminative ability can be combined to represent and localize the target. The combination of features should be carried out carefully. In an intuition, the more feature we use, the better of the tracking performance. However, this is not true in practice. According to information theory, the feature added into the system can bring negative effect as well as improvement of the performance [7]. This is due to the fact that the features used are not totally independent. Instead, they are correlated.

In our implementation, two kinds of features are used to represent the target. According to the experimental results, this number is appropriate in most cases. We have tested a system by using 1 or 3 features, which gave worse performance. During the initialization of the tracker, the features ranked in top two are selected for the tracking. The feature selection module runs every 8 to 12 frames. When the feature selection module selects features different from those in the initialization, only one feature is replaced once. Only the second feature of the previous selection will be discarded and replaced by the best one in current selection. This strategy is very important in keeping the target from drifting.

\section{Target Localization in the Adaptive Tracking}

The proposed tracking algorithm combines the top two features through the back-projection [2] of the joint histogram. Based on Equation(4), we calculate a joint histogram the target with the top two features,

$p_{f}^{\left(b_{i n}^{(1)}, b_{i n}^{(2)}\right)}=C \sum_{\mathbf{x}_{i} \in R_{f}} k\left(\left\|\mathbf{x}_{i}\right\|\right) \delta\left[h\left(\mathbf{x}_{i}\right)-b_{i n}^{(1)}\right] \delta\left[h\left(\mathbf{x}_{i}\right)-b_{i n}^{(2)}\right]$

and a joint histogram of the searching region

$p_{b}^{\left(b_{i n}^{(1)}, b_{i n}^{(2)}\right)}=C \sum_{\mathbf{x}_{i} \in R_{b}} k\left(\left\|\mathbf{x}_{i}\right\|\right) \delta\left[h\left(\mathbf{x}_{i}\right)-b_{i n}^{(1)}\right] \delta\left[h\left(\mathbf{x}_{i}\right)-b_{i n}^{(2)}\right]$.

We get a division histogram by dividing the joint histogram of the target by the joint histogram of the background,

$$
p_{d}^{\left(b_{i n}^{(1)}, b_{i n}^{(2)}\right)}=\frac{p_{f}^{\left(b_{i n}^{(1)}, b_{i n}^{(2)}\right)}}{p_{b}^{\left(b_{i n}^{(1)}, b_{i n}^{(2)}\right)}} .
$$

The division histogram is normalized for the histogram back-projection. The pixel values in the image are associated with the value of the corresponding histogram bin by histogram back-projection. The back-projection of the target histogram with any consecutive frame generates a probability image $p=\left\{p_{w}^{i}\right\}_{i=1 \ldots n_{h}}$ where the value of each pixel characterizes the probability that the input pixel belongs to the histograms. The two images of the top two features have been computed for the back-projection. Note, the H, S, $r$, and $g$ images are calculated by transfer the original image to the HSV space and the $r g$ space; the orientation image has been calculated by using the approach introduced in section II(B).

Since we are using Epanechnikov profile, the derivative of the profile, $g(x)$, is constant. The target's shift vector in the current frame is computed as

$$
\hat{\mathbf{y}}_{1}=\frac{\sum_{i=1}^{n_{h}} \mathbf{x}_{i} p_{w}^{i}}{\sum_{i=1}^{n_{h}} p_{w}^{i}} .
$$

The tracker assigns a new position to the target by using

$$
\hat{\mathbf{y}}_{1}=\frac{1}{2}\left(\hat{\mathbf{y}}_{0}+\hat{\mathbf{y}}_{1}\right) \text {. }
$$

If $\left\|\hat{\mathbf{y}}_{0}-\hat{\mathbf{y}}_{1}\right\|<\varepsilon$, this position is assigned to the target. Otherwise, compute the Equation(19) again. In our algorithm, the number of the computation is set to less than 15. In most cases, the algorithm converges in 3 to 6 loops.

\section{Experimental Results}

To check the effectiveness of the proposed method, we have implemented and tested on a wide variety of challenging image sequences with different environments and applications. The tracking results are compared with those given by the basic mean shift algorithm. The current implementation ran 14 frames/sec on an Intel Centrino $1.4 \mathrm{GHz}$ laptop with $256 \mathrm{MB}$ RAM when applied to images of size $640 \times 480$. It achieved 32 frames/sec on an Intel Pentium D 3.0GHz PC with 1GB RAM.

Pedestrian tracking is very important in visual surveillance. The first tracking example (Fig.2) uses a video sequence captured in a room ${ }^{1}$. The illumination changes during the tracking process. The color on the human changes very much in these images. Our method adapts the model in the tracking process and successfully tracks the whole sequence. In contrast, the basic mean shift algorithm fails very quickly.

The second tracking example (Fig.3) also uses a pedestrian sequence from the same source. However, this tracking task is more challenging because the color of the man's clothes is very similar to that of the floor. The standard mean shift algorithm fails quickly at the third frame. In contrast, our method selects shape cue as discriminative feature at the very beginning and tracks the man successfully until the man becomes too small to be tracked. This example demonstrates that the shape cue is very important and should not be omitted.

\footnotetext{
${ }^{1}$ This image sequence was captured in an entrance lobby. It can be obtained from http://groups.inf.ed.ac.uk/vision/caviar/.
} 


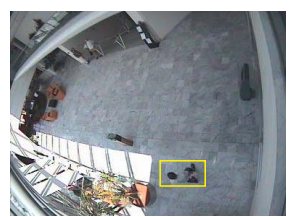

(a)

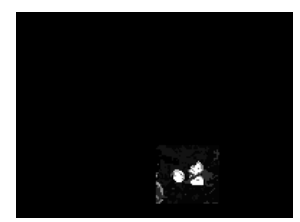

(f)

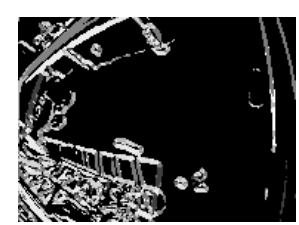

(b)

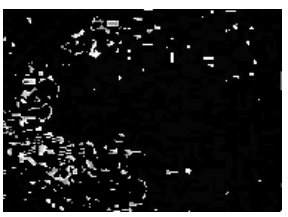

(g)

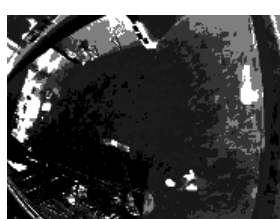

(c)

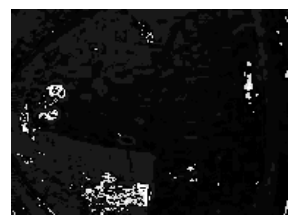

(h)

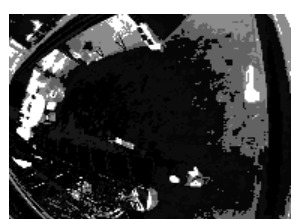

(d)

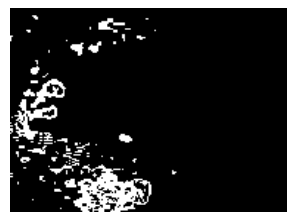

(i)

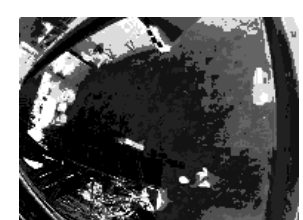

(e)

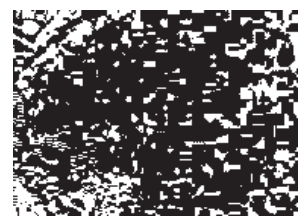

(j)

Fig. 1. (a) Original image. Likelihood ratio image of (b) orientation histogram; (c) R; (d) G; (e) B; (f) integration of the top two features: orientation histogram and R; (g) H; (h) S; (i) r; and (j) g. Note that the likelihood image in (f) only shows the neighborhood of the target.

Face tracking is crucial for the new generation of $\mathrm{Hu}-$ man and Computer Interface (HCI). The video sequence ${ }^{2}$ containing faces are tested using our method (Fig.4). The man's face moves from the left to right very quickly and back versa. The illumination on the face is varying in the sequence. There are clutters and ambiguous colors in the background. The basic mean shift algorithm fails at the $5^{\text {th }}$ frame. The proposed method tracks the faces successfully in the whole sequence.

The above tests have shown that no single cue is always discriminative enough against the background, which is crucial for tracking. The proposed tracker which can select good cues is the key to successful tracking in those sequences.

We found that the best features selected in the initialization stage are very important. The shape of the target is very accurate during that stage and there is little ambiguity between background and the target. These features should not be abandoned easily. The strategy described in section $\mathrm{IV}(\mathrm{B})$ is effective in our experiments.

\section{Conclusion}

We present an adaptive tracking algorithm by integrating the shape and color features in an adaptive way. The proposed algorithm has been tested on many sequences and proved efficient and reliable. In comparison with the standard mean shift algorithm, our approach provides much better results.

\section{REFERENCES}

[1] S. Birchfield, "Elliptical Head Tracking using Intensity Gradients and Color Histograms", in Proc. of the IEEE Conf. on Computer Vision and Pattern Recognition, pp. 232-237, 1998.

[2] G.R. Bradski, "Computer Vision Face Tracking as a Component of a Perceptural User Interface," in Proc. of the IEEE Workshop Applications of Computer Vision, pp. 214-219, 1998.

[3] M.J. Black and A.D. Jepson, "EigenTracking: Robust Matching and Tracking of Articulated Objects using a View-based Representation," Int'l Journal of Computer Vision, vol. 26, no.1, pp.63-84, 1998.

\footnotetext{
${ }^{2}$ The human face image sequence can be obtained from http://vision.stanford.edu/ birch/headtracker/.
}

[4] R. T. Collins and Y. Liu, "On-line Selection of Discriminative Tracking Features," in IEEE Trans. on Pattern Analysis and Machine Intelligence, Vol. 27, No. 10, October 2005.

[5] D. Comaniciu and P. Meer, "Mean Shift: A Robust Approach Toward Feature Space Analysis," IEEE Trans. on Pattern Analysis and Machine Intelligence, vol. 24, no. 5, pp. 603-619, 2002.

[6] D. Comaniciu, V. Ramesh, and P. Meer, "Kernel-based Object Tracking," IEEE Trans. Pattern Analysis Machine Intelligence, vol. 25, no. 5, pp. 564-577, 2003.

[7] T.M Cover and J.A. Thomas, Elements of Information Theory, John Wiley and Sons Press, 1991.

[8] A. Djouadi, O. Snorrason, and F. Garber, "The Quality of Training sample Estimates of the Bhattacharyya Coefficient," IEEE Trans. on Pattern Analysis and Machine Intelligence, vol. 12, pp.92-97, 1990.

[9] T. Drummond and R. Cipolla, "Real-time Visual Tracking of Complex Structures," IEEE Trans. on Pattern Analysis and Machine Intelligence, vol. 4, no. 7, pp. 932-946, 2002.

[10] H. Stern and B. Efros, "Adaptive Color Space Switching for Face Tracking in Multi-color Lighting Environment," in Proc. IEEE Int'l Conf. on Automatice Face and Gesture Recognition. Washington DC, USA, 2002, pp. 249-254.

[11] D. Koller, K. Daniilidis, T. Thorhallson, and H.-H. Nagel, "Modelbased Object Tracking in Traffic Scenes," in Proc. European Conf. on Computer Vision, pp. 437-452, 1992.

[12] C. Olson, "Maximum-Likelihood Template Tracking," in Proc. of the IEEE Conf. on Computer Vision and Pattern Recognition, vol. 2, pp. 52-57, 2000.

[13] J. Shi and C. Tomasi, "Good Features to Track," in Proc. of the IEEE Conf. on Computer Vision and Pattern Recognition, pp. 717723, 1997.

[14] H. Sidenbladh, M.J. Black, and D.J. Fleet, "Stochastic Tracking of 3D Human Figures using 2D Image Motion," in European Conf. on Computer Vision, vol. 2, pp. 702-718, 2000.

[15] M. Swain and D. Ballard, "Color Indexing," Int'l Journal of Computer Vision, vol. 7, pp. 11-32, 1991.

[16] H. Tao, H.S. Sayhney, and R. Kumar, "Dynamic Layer Representation with Applications to Tracking," in Proc. of the IEEE Conf. on Computer Vision and Patter Recognition, vol. 2, pp. 134-141, 2000.

[17] M. Vincze, M. Schlemmer, P. Gemeiner, and M. Ayromlou, "Vision for Robotics: a tool for model-based object tracking," IEEE Trans. on Robotics, vol. 12, no. 4, pp. 53-64, 2005.

[18] S. Wachter and H. Nagel, "Tracking Persons in Monocular Image Sequences," Computer Vision and Image Understanding, vol. 74, no.3, pp. 174-192, 1999. 

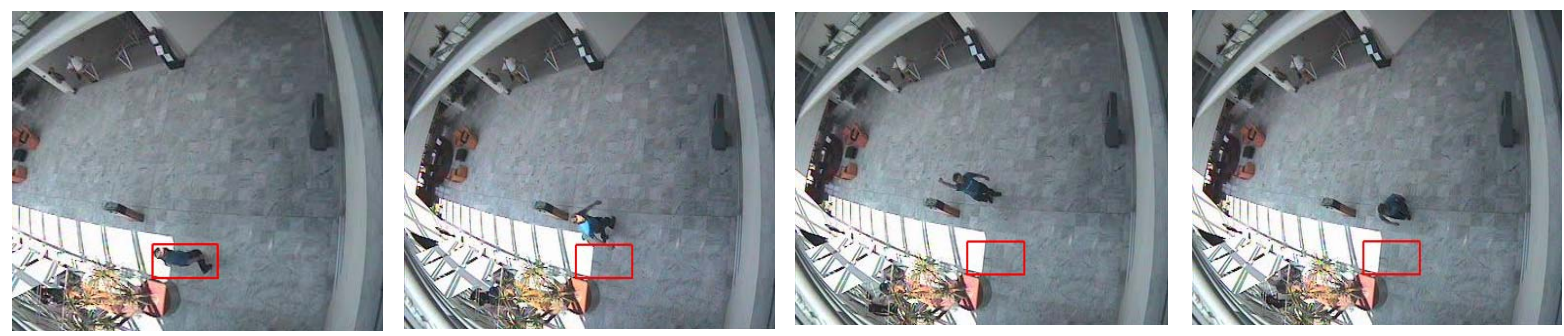

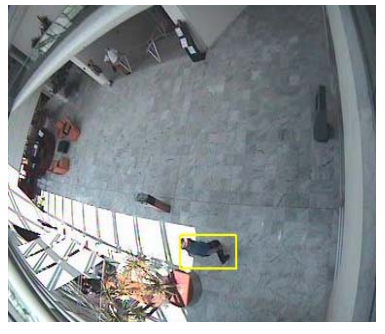

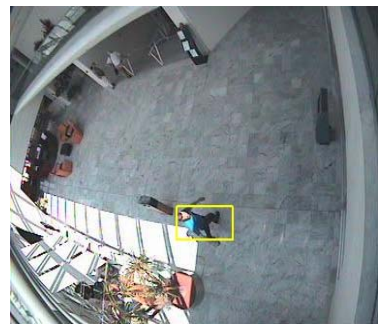

f10

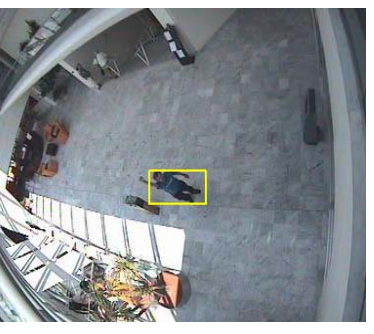

130

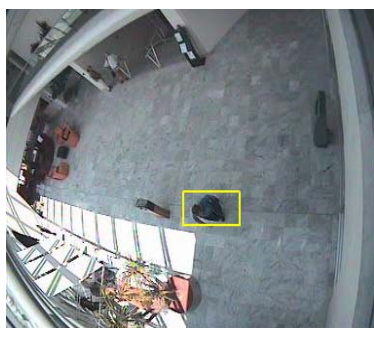

f70

Fig. 2. Tracking results using the basic mean shift algorithm (in the first row) and the proposed method (in the second row) of a pedestrian in an indoor environment.

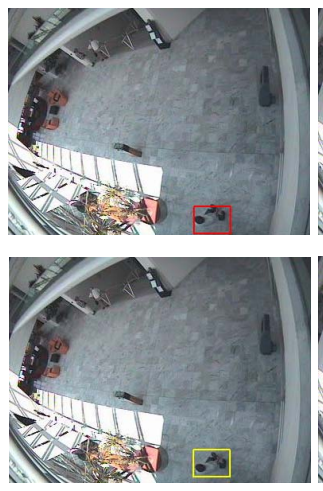

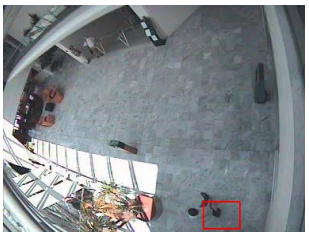
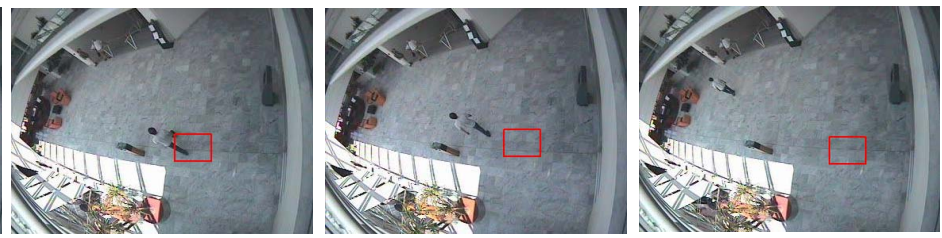

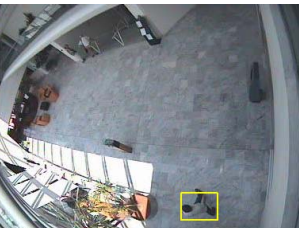

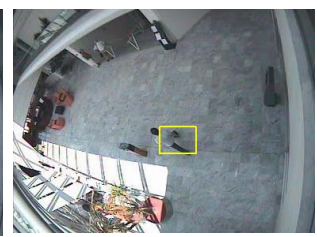

f43

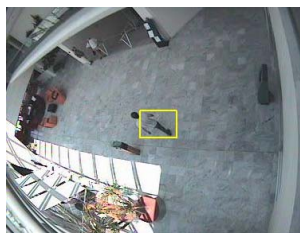

553

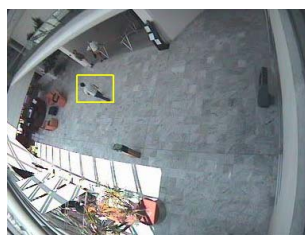

f100

Fig. 3. Tracking results using the basic mean shift algorithm (in the first row) and the proposed method (in the second row) of another person. The color of the target is similar to the background.
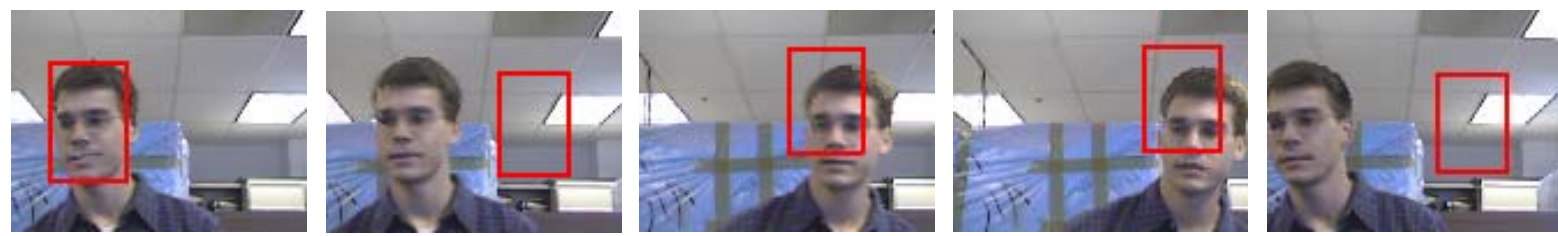

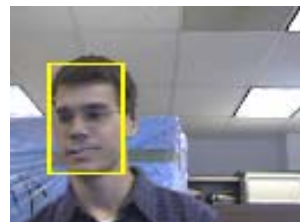

f1

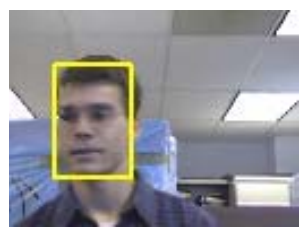

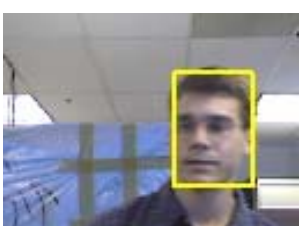

$\mathrm{f12}$

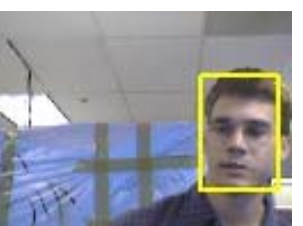

$\mathrm{f} 20$

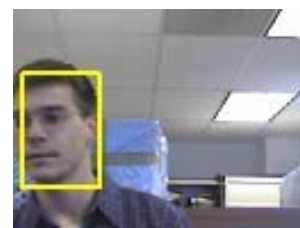

f25

Fig. 4. Face tracking results using the basic mean shift algorithm (in the first row) and the proposed method (in the second row). The face in the sequence moves quickly. 